\section{Padrão de atividade física entre gestantes atendidas pela estratégia saúde da família de Campina Grande - PB}

\section{Physical activity patterns in pregnant women attending the family health program of Campina Grande - $P B$}

Jousilene de Sales Tavares ${ }^{1}$

Adriana Suely de Oliveira Melo ${ }^{1,2}$

Melania Maria Ramos de Amorim ${ }^{2,3}$

Vivianne Oliveira Barros'

Mônica Yuri Takito ${ }^{4}$

Maria Helena D’Aquino Benício ${ }^{4}$

Maria Aparecida Alves Cardoso'

${ }^{1}$ Núcleo de Estudos e Pesquisas Epidemiológicas (NEPE)/ Universidade Estadual da Paraíba

${ }^{2}$ Instituto Materno Infantil Professor Fernando Figueira (IMIP)

${ }^{3}$ Faculdade de Medicina/Universidade Federal de Campina Grande

${ }^{4}$ Universidade de São Paulo

Financiamento: CNPq - Edital CT - Saúde - n 30/2004

Edital Alimentação, Nutrição e Promoção da Alimentação e Modos de Vida Saudáveis - nº 051/2005 Correspondência: Jousilene de Sales Tavares. R. Arruda Câmara, 626 - Santo Antônio - Campina Grande, PB CEP 58103-273. E-mail: tenjousilene@gmail.com

\section{Resumo}

Introdução: A atividade física realizada durante a gestação vem sendo discutida devido aos seus efeitos benéficos tanto para a saúde materna como para o crescimento fetal e desfechos gestacionais, porém ainda são escassos estudos sobre o padrão de atividade física neste período. Objetivo: Avaliar o padrão de atividade física entre gestantes atendidas pela estratégia Saúde da Família (ESF) do município de Campina Grande/ PB. Métodos: Foi acompanhada uma coorte observacional de gestantes $(\mathrm{n}=118)$, a partir da $16^{a}$ semana gestacional, durante os anos de 2005 a 2006. A cada quatro semanas eram aferidas as condições clínicas, socioeconômicas e obstétricas, incluindo a aplicação de um questionário específico sobre atividade física na 16ª $24^{\mathrm{a}}$ e $32^{\mathrm{a}}$ semanas gestacionais. A avaliação foi feita a partir da somatória do equivalente metabólico (METs) e as atividades cotidianas foram divididas em quatro grupos: atividades laboral, doméstica e caminhada, além de inatividade. As gestantes foram classificadas de acordo com o padrão de atividade física realizado em: sedentárias, praticantes de atividades física leve, moderada e vigorosa. Os dados foram analisados no programa Epi Info 3.4.1. Resultados: As características socioeconômicas da coorte estudada indicaram majoritariamente gestantes de baixo poder aquisitivo, baixa escolaridade e baixo percentual de mulheres economicamente ativas. $\mathrm{O}$ padrão de atividade física observado foi baixo desde o primeiro trimestre gestacional, oscilando entre o leve e o sedentário, e foi diminuindo com o evoluir da gravidez, com $100 \%$ das gestantes alcançando o padrão sedentário na $32^{\mathrm{a}}$ semana. Em relação aos grupos de atividades, observou-se um predomínio de atividades domésticas, seguidas pelas atividades de lazer. Conclusão: Na coorte estudada verificou-se um padrão de atividade física inadequado desde o início da gestação, agravando-se no terceiro trimestre gestacional.

Palavras-chaves: Atividade Motora. Gestação. MET. 


\section{Abstract}

Introduction: The benefits of physical activity during pregnancy on fetal growth, maternal health and pregnancy outcomes have been debated; however, studies on the physical activity patterns during this period are still scarce. Objective: To evaluate the physical activity patterns of pregnant women attending the Family Health Strategy at the municipality of Campina Grande, PB. Methods: A cohort of 118 pregnant women was followed from 2005 to 2006 . Follow up started on the $16^{\text {th }}$ gestational week and continued at every four weeks to assess clinical conditions and collect obstetric and socio-economic information. A specific questionnaire on physical activity was applied on the $16^{\text {th }}, 24^{\text {th }}$ and $32^{\text {nd }}$ gestational weeks, which was estimated through the weekly sum of the metabolic equivalent (MET). Daily activities were classified in four groups: labor, household, walking, and inactivity. According to the physical activity pattern, women were classified as sedentary, or performing light, moderate or vigorous activity. The data set was analyzed in Epi Info 3.4.1 Results: The socioeconomic characteristics of the cohort described a population of low levels of income and education. The physical activity pattern observed since the first gestational trimester was low, ranging from light to sedentary and it decreased along pregnancy. On the $32^{\text {nd }}$ gestational week $100 \%$ of the women were sedentary. Regarding physical activity groups, women spent more time on household activities followed by recreational activities. Conclusion: The physical activity pattern observed was inadequate form the beginning of pregnancy and it worsened in the third gestational trimester.

Keywords: Physical activity. Pregnancy. MET.

\section{Introdução}

A prática da atividade física durante a gestação vem sendo recentemente foco de debates na comunidade cientifica. Além de exercer influência sobre o ganho ponderal materno e o crescimento fetal, a sua prática está associada à prevenção e ao controle de diversas doenças, como diabetes e da préeclâmpsia, proporcionando efeitos benéficos em desfechos gestacionais ${ }^{1-4}$.

Entende-se por atividade física qualquer movimento corporal produzido pelos músculos esqueléticos que resulte em gasto energético maior que os níveis de repouso ${ }^{5}$, incluindo não apenas o exercício físico (atividade física planejada, estruturada, acompanhada e realizada de forma sistemática, com o objetivo de melhorar ou manter a aptidão física), como também as atividades cotidianas, laborais e de lazer.

O método mais utilizado para avaliar a intensidade da atividade física é a determinação do MET (medidor de estimativa de gasto calórico total), correspondendo à quantidade mínima necessária de oxigênio para as funções metabólicas do organismo, sendo considerado 1 MET a taxa de repouso metabólico obtida durante o estado de repouso total ${ }^{6}$. De acordo com um compêndio de atividade física publicado em $2000^{6}$, o nível dos METs pode variar de 0,9 durante o sono a 18 durante uma corrida (5,5 milhas/ $\min )$.

A partir da determinação dos METs das atividades físicas, utilizando questionário validado para gestantes, Chasan-Taber et al. ${ }^{7}$ assim classificaram as gestantes de acordo com a intensidade do padrão de atividade física: sedentária (METs $<1,5$ ), leve (METs de 1,5 a 2,9), moderada (METs de 3 a 5,9) e vigorosa (METs $\geq 6$ ).

Tanto estudos nacionais como internacionais indicam que o padrão de atividade física das gestantes oscila entre sedentário e leve $\mathrm{e}^{8-10}$. Além de observar este mesmo padrão, um estudo de coorte realizado na região Sudeste do Brasil também identificou baixa prevalência de atividade física e de lazer entre as gestantes ${ }^{11}$. 
O estudo visa avaliar o padrão de atividade física entre gestantes atendidas pela Estratégia Saúde da Família no município de Campina Grande-PB, destacando as atividades laboral, doméstica, caminhada e inatividade.

\section{Métodos}

As gestantes estudadas faziam parte dos projetos "Impacto da atividade física e da orientação alimentar durante a gestação sobre o ganho de peso gestacional e desfechos da gravidez" e "Impacto da orientação alimentar e da atividade física sobre o fluxo sanguíneo feto-placentário e crescimento fetal", coordenados, respectivamente, pelo Núcleo de pesquisa em nutrição e saúde (NUPENS/USP) e pelo Núcleo de estudos epidemiológicos (NEPE/UEPB). Uma coorte com amostra de 137 gestantes que iniciaram pré-natal na Estratégia Saúde da Família do município de Campina Grande - PB foi acompanhada desde o início da gravidez até o parto; o seguimento durou treze meses, de março de 2005 a março de 2006. A pesquisa foi aprovada pelo Comitê de Ética da Universidade de São Paulo e da Universidade Estadual da Paraíba, e seguiu as diretrizes éticas de pesquisa com seres humanos, resolução 196/96 do Conselho Nacional de Saúde.

A amostra foi calculada no programa OpenEpi versão 2, com base na prevalência de sedentarismo no primeiro trimestre da gestação em um estudo realizado em Fortaleza $^{12}$. A prevalência foi estimada em $28,9 \%$, resultando em um tamanho amostral de 119 gestantes, com poder de $80 \%$ e erro alfa de 0,05 .

As gestantes eram convidadas para participar da pesquisa e, caso concordassem, assinavam o termo de consentimento livre e esclarecido. Foram critérios de inclusão: idade igual ou superior a 18 anos, idade gestacional menor ou igual a 16 semanas, gestação única e feto vivo. Excluíram-se as gestantes com as seguintes condições: doenças maternas crônicas, malformações fetais, perdas fetais, parto prematuro antes da $32^{a}$ semana e gestantes incapacitadas de desenvolverem atividades físicas habituais (Figura 1).

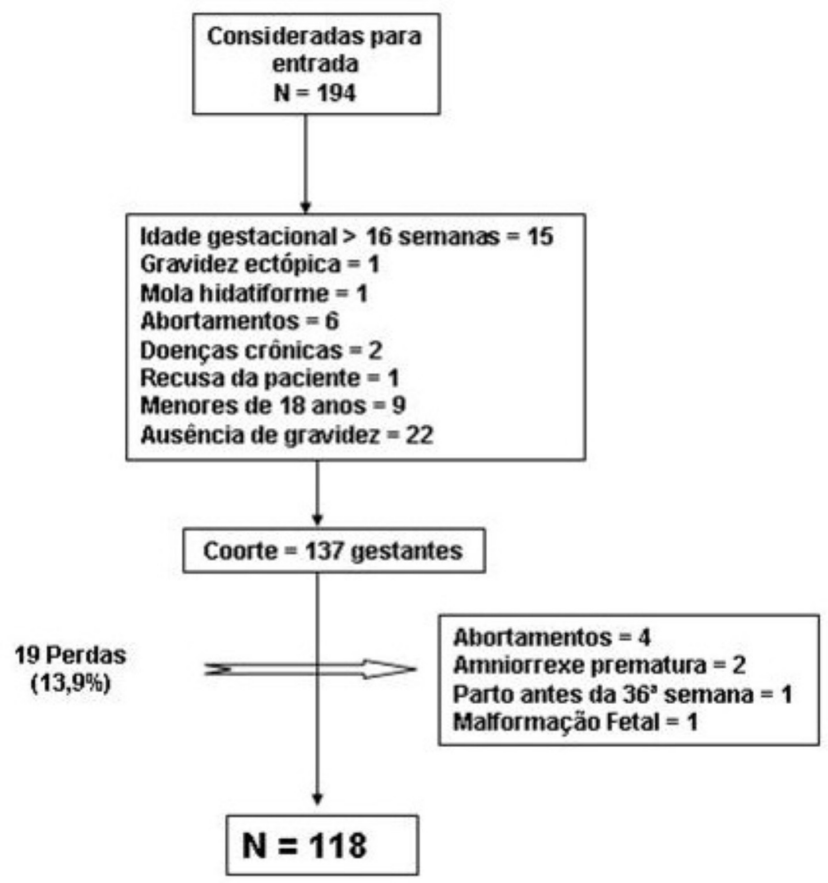

Figura 1 - Organograma das exclusões e perdas da coorte Figure 1 - Chart of the exclusions and losses in the cohort 
As gestantes foram inicialmente avaliadas na $16^{\text {a }}$ semana, período em que responderam a questionários abordando informações obstétricas, socioeconômicas e clínicas. Um questionário específico semi-estruturado, desenvolvido e validado para gestantes brasileiras por Takito et al ${ }^{13}$ foi aplicado em três momentos, na 16 ${ }^{\mathrm{a}}, 24^{\mathrm{a}}$ e $32^{\text {a }}$ semanas gestacionais, para avaliar o padrão de atividade física cotidiano.

A atividade física foi medida através do cálculo do "MET" referente às atividades relatadas pelas gestantes nos questionários específicos ${ }^{11}$. Uma variável denominada METgest, foi criada para estimar o padrão de atividade física da coorte. Inicialmente foi obtida uma estimativa da freqüência (semanal) e duração (em minutos) das atividades cotidianas. A seguir, multiplicouse a freqüência de cada atividade por sua duração em minutos, obtendo-se um total de tempo em uma semana (TTS) gasto pela gestante com essa atividade. Para elaborar o estimador, aqui denominado METgest, o TTS de cada atividade foi multiplicado pelo MET referente a essa atividade, de acordo com o compêndio de atividade física ${ }^{6}$, obtendo-se um TTS ponderado pelo MET para cada atividade (TTSP). O METgest, então, foi calculado dividindo-se o somatório ponderado de TTSs $\left(\Sigma_{\text {TTSP }}\right)$ pelo somatório de tempo das atividades em uma semana $\left(\Sigma_{\text {TTS }}\right)$. Para este cálculo foi utilizada a seguinte fórmula:

\section{METgest $=\sum_{\mathrm{TTSP}} / \sum_{\mathrm{TTS}}$}

Onde: $\operatorname{TTSP}_{\mathrm{i}}=\mathrm{TTS}_{\mathrm{i}} \times \mathrm{MET}_{\mathrm{i}}$ $\mathrm{TTS}_{\mathrm{i}}=\mathrm{F}_{\mathrm{i}} \mathrm{xT}_{\mathrm{i}}$ $\mathrm{F}=$ freqüência de cada atividade

$\mathrm{T}=$ tempo gasto com cada atividade

$\mathrm{i}=$ índice i para cada atividade cotidiana

TTSP = freqüência de cada atividade pela sua duração em minutos multiplicada pelo índice da atividade

TTS = freqüência de cada atividade pela sua duração em minutos

As gestantes foram classificadas de acordo com os níveis de intensidade de ME-
Tgest, método adaptado de Chasan-Taber et $\mathrm{al}^{7}$. Assim, valores de METgest menores que 1,5 classificam a gestante como sedentária; entre 1,5 a 2,9 equivale ao padrão de atividade física leve; entre 3 e 5,9 o padrão é considerado moderado, e acima de 5,9 vigoroso.

O somatório ponderado de TTSPs para cada atividade estudada foi separado em quatro grupos de acordo com a característica da atividade: laboral, doméstica, caminhada e inatividade. Foram consideradas inatividade: assistir televisão e descansar. Já o grupo de atividades domésticas contemplou: limpeza leve e pesada, lavar, estender e passar roupa, cozinhar, lavar louça, varrer casa, quintal, cuidar de crianças e limpar tapetes. No grupo da caminhada foram consideradas todas as caminhadas realizadas pelas gestantes, com o intuito de realizar exercício ou por fazer parte das suas obrigações cotidianas, como, por exemplo, levar criança à escola, fazer compras, ir para o trabalho, entre outras. Consistiram atividades laborais todas aquelas realizadas no trabalho: andar, carregar objetos pesados, ficar em pé parada e sentada.

O programa Epi Info versão 3.4.1 foi utilizado para a análise estatística. Realizouse inicialmente uma análise descritiva das variáveis demográficas, socioeconômicas, obstétricas, tabagismo, prática de esportes e padrão de atividade física. As diferenças no padrão de atividade física entre os diferentes momentos da gravidez foram avaliadas através da comparação de médias e desvio-padrão dos METs, na $16^{\mathrm{a}}, 24^{\mathrm{a}}$ e $32^{\mathrm{a}}$ semanas. Os grupos de atividade física foram comparados entre si e com a evolução da gestação através de análise de variância. Admitiu-se um erro alfa de 0,05.

\section{Resultados}

Dentre as 137 gestantes estudadas ocorreram $16,5 \%$ de perdas de seguimento, resultando em uma amostra de 118 . Não houve diferença estatisticamente significante no padrão de atividade física observado no início do seguimento entre 
as gestantes incluídas no estudo e as perdas registradas.

A idade materna média foi de 24,3 anos, variando de 18 a 43 anos. A média de anos de estudo da coorte foi de sete anos, variando de 0 a 12 anos, e a renda familiar per capita média foi de 161,70 reais, variando de 0 a 666,00 reais. A maioria das gestantes eram multigestas $(66,9 \%)$, apresentando um intervalo interpatal em sua maioria entre dois e cinco anos com $39,7 \%$. O percentual das gestantes que trabalhavam fora de casa foi de $34,7 \%$, e $91,5 \%$ das gestantes não faziam uso do fumo (dados coletados na $16^{\mathrm{a}}$ semana)(Tabela 1).

As diferenças no padrão de atividade física das gestantes nos pontos de seguimento $16^{\mathrm{a}}$, 24a e $32^{\mathrm{a}}$ semanas gestacionais podem ser visualizadas na Figura 2. Houve um predomínio de atividade física leve na $16^{\text {a }}$ semana, com cerca de $85 \%$ das gestantes sendo classificadas nesta categoria. $\mathrm{Na} 24^{\mathrm{a}}$

Tabela 1 - Distribuição das gestantes de acordo com as variáveis demográfica, socioeconômicas, obstétricas e tabagismo. Campina Grande-PB, 2005 e 2006.

Table 1 - Distribution of pregnant women according to demographic, socioeconomic, obstetric variables and smoking. Campina Grande-PB, 2005 and 2006.

\begin{tabular}{|c|c|c|}
\hline Variáveis & $\mathrm{n}$ & $\%$ \\
\hline \multicolumn{3}{|l|}{ Idade materna (em anos) } \\
\hline 18 a 20 & 28 & 23,7 \\
\hline$>20$ & 90 & 76,3 \\
\hline \multicolumn{3}{|l|}{ Escolaridade } \\
\hline$<8$ anos & 77 & 65,3 \\
\hline$>8$ anos & 41 & 34,7 \\
\hline \multicolumn{3}{|l|}{ Número de gestações } \\
\hline Primigesta & 39 & 33,1 \\
\hline Multigesta & 79 & 66,9 \\
\hline \multicolumn{3}{|c|}{ Intervalo interpartal (em anos) } \\
\hline$<2$ & 21 & 27 \\
\hline$\geq 2$ e $\leq 5$ & 31 & 39,7 \\
\hline$>5$ & 26 & 33,3 \\
\hline \multicolumn{3}{|c|}{ Número de consultas pré-natais } \\
\hline$<6$ & 15 & 12,9 \\
\hline$\geq 6$ & 101 & 87,1 \\
\hline \multicolumn{3}{|l|}{ Trabalho materno } \\
\hline Sim & 41 & 34,7 \\
\hline Não & 77 & 65,3 \\
\hline \multicolumn{3}{|l|}{ Renda familiar per capita } \\
\hline Até $\mathrm{R} \$ 75,00$ & 32 & 27,4 \\
\hline De $R \$ 76,00$ a 150,00 & 41 & 35 \\
\hline De $R \$ 151,00$ a 225,00 & 19 & 16,2 \\
\hline De $\mathrm{R} \$ 226,00$ a 300,00 & 15 & 12,8 \\
\hline Acima de $R \$ 300,00$ & 10 & 8,5 \\
\hline \multicolumn{3}{|l|}{ Tabagismo } \\
\hline Sim & 10 & 8,5 \\
\hline Não & 108 & 91,5 \\
\hline
\end{tabular}


semana quase $100 \%$ das gestantes foram consideradas sedentárias, e este padrão atingiu todas as gestantes no final do seguimento.

Avaliando-se as médias de atividade física para as semanas correspondentes aos trimestres gestacionais, observa-se uma redução da atividade ao longo do período gestacional $(p<0,01)$ (Figura 3). Na 16 a semana a média dos METs observada foi de 1,9 $\pm 0,38$ (variando de 0,86 a 2,97), reduzindo para $0,68 \pm 0,13$ (variando de 0,35 a 3,00 ) na $24^{\text {a }}$ semana e alcançando 0,58 $\pm 0,13$ (variando de 0,31 a 1) na $32^{\text {a }}$ semana.

Dentre os grupos de atividade estudados, verificou-se uma maior média do TTSP para as atividades domésticas, sendo seguidas pelas médias das atividades laborais e caminhada, registrando-se uma diferença estatisticamente significante

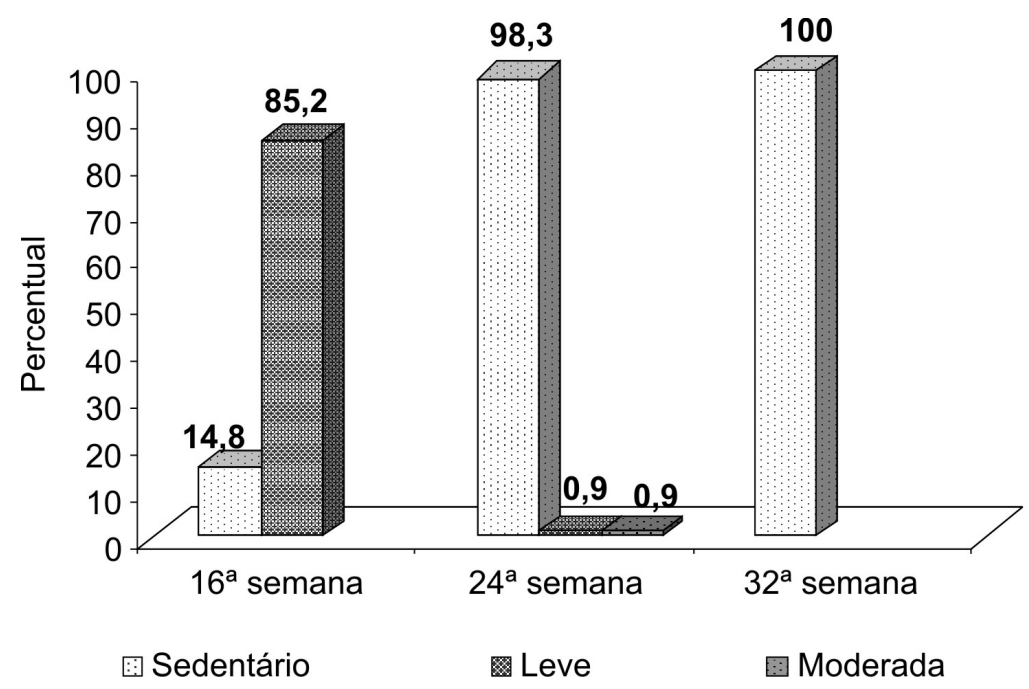

Figura 2 - Descrição do Padrão de Atividade Física das gestantes nos pontos estudados. Campina Grande 2005 e 2006.

Figure 2 - Description of Physical Activity Patterns of pregnant women at the times points studied. Campina Grande 2005 and 2006.

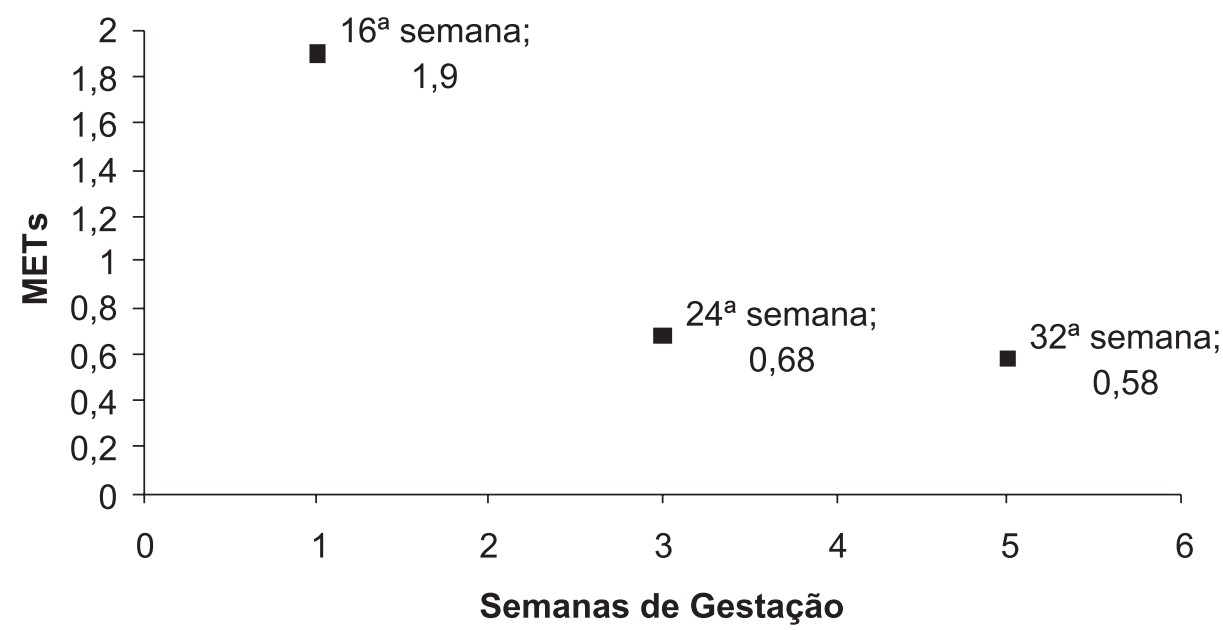

Figura 3 - Média dos METs de acordo com a idade gestacional estudada. Campina Grande-PB, 2005 e 2006.

Figure 3 - Average METs according to gestational age. Campina Grande-PB, 2005 and 2006. 
Tabela 2 - Média e desvio-padrão do TTSP das atividades cotidianas de acordo com o grupo de atividade. Campina Grande-PB, 2005 e 2006.

Table 2 - Average and standard deviation of TTSP (weighed total weekly time) of every-day activities according to the group of activity. Campina Grande-PB, 2005 e 2006.

\begin{tabular}{lccccc}
\hline & $\begin{array}{c}\text { Inatividade } \\
\text { TTSP } \\
\text { Média } \pm \text { DP }\end{array}$ & $\begin{array}{c}\text { Doméstica } \\
\text { TTSP } \\
\text { Média } \pm \text { DP }\end{array}$ & $\begin{array}{c}\text { Laboral } \\
\text { TTSP } \\
\text { Média } \pm \text { DP }\end{array}$ & $\begin{array}{c}\text { Caminhada } \\
\text { TTSP } \\
\text { Média } \pm \text { DP }\end{array}$ & $p$ \\
\hline 16a semana & $692,61 \pm 517,47$ & $420,54 \pm 286,33$ & $231,70 \pm 478,12$ & $110,13 \pm 135,10$ & $<0,001$ \\
24a semana & $614,46 \pm 501,26$ & $337,23 \pm 220,51$ & $271,41 \pm 555,23$ & $82,65 \pm 120,06$ & $<0,001$ \\
32a semana & $658,64 \pm 502,03$ & $326,66 \pm 186,48$ & $251,33 \pm 551,35$ & $89,06 \pm 97,50$ & $<0,001$ \\
$P$ & 0,49 & $<0,001$ & 0,85 & 0,17 & \\
\hline
\end{tabular}

entre os grupos. Comparando-se os quatro grupos, incluindo o grupo representado pela inatividade física, este último apresentou maior média.

Ao se analisar as atividades com o evoluir da gestação, evidenciou-se uma queda sensível do TTSP das atividades domésticas $(p<0,001)$. Não foi observada diferença estatisticamente significante em relação ao grupo de atividades de lazer e laboral ( $p=0,49 \mathrm{e}$ 0,85 , respectivamente). Em relação ao grupo de caminhada, não se observou diferença estatisticamente significante quando comparados os três pontos de estudo, porém observou-se uma tendência de queda após a $16^{\text {a }}$ semana (Tabela 2 ). Dentre as atividades que representam a inatividade física destacam-se assistir televisão, como sendo o principal constituinte do TTSP deste grupo (dados não apresentados em tabela).

\section{Discussão}

Em termos de condições socioeconômicas, a coorte caracteriza-se pelo baixo poder aquisitivo, baixa escolaridade e baixo percentual de mulheres economicamente ativas, o que era esperado por se tratar de população advinda majoritariamente das áreas mais carentes da cidade.

$\mathrm{Na} 16^{\text {a }}$ semana, a categoria de atividade leve predominou como padrão de atividade física da coorte; neste momento, a maioria das gestantes foi classificada como praticante de leve intensidade e já se observa uma proporção de $15 \%$ de gestantes sedentárias. Nenhuma gestante apresentou padrão moderado ou vigoroso. Resultado similar foi encontrado em estudo de coorte realizado no Rio de Janeiro por Bicalho ${ }^{10}$, que verificou uma prevalência de $98,1 \%$ de gestantes sedentárias, não tendo sido observada nenhuma gestante praticante de atividade física moderada.

Com a evolução da gestação, observouse que o padrão de atividade física foi sendo ainda mais modificado e reduzido. O próximo ponto do seguimento indicou que a quase totalidade das gestantes passou ao nível sedentário do final do primeiro para o segundo trimestre gestacional. $\mathrm{Na} 32^{\mathrm{a}}$ semana, todas as gestantes foram classificadas como sedentárias. Este fato merece considerações tanto de ordem biológica e fisiológica como cultural. O próprio peso e a mudança do centro de gravidade fazem com que as gestantes se sintam menos dispostas ao final da gestação ${ }^{14}$. Por outro lado, sabese que até pouco tempo, a recomendação geral para as gestantes era a redução das atividades ou até mesmo o repouso ${ }^{15}$. Este "cuidado" ainda parece fazer parte do senso comum, segundo o qual toda e qualquer gestante deve reduzir a atividade física, devendo repousar mais com a evolução da gravidez. Corroborando estes dados, um estudo realizado por Silva et al. ${ }^{12} \mathrm{em}$ Fortaleza, avaliando 305 gestantes, também observou uma acentuada redução no padrão de atividade física com a evolução da gravidez. Rousham et al. ${ }^{16}$, acompanhando 57 gestantes no Reino Unido, avaliaram a atividade 
física destas mulheres na $12^{\mathrm{a}}, 16^{\mathrm{a}}, 25^{\mathrm{a}}, 34^{\mathrm{a}} \mathrm{e}$ $38^{\mathrm{a}}$ semana de gestação. Estes autores também observaram uma redução dos METs com a evolução da gestação, passando de 1,51 no início da gestação para 1,29 na sua fase final. No presente estudo esta redução foi bem mais evidente, alcançando 0,58 na $32^{\text {a }}$ semana.

Além da redução do padrão com a evolução da gravidez, que per se já é considerado um agravante para um estilo de vida saudável, percebemos na coorte estudada uma baixa prevalência de um padrão considerado ideal de atividade física nos três pontos estudados, que seria o padrão moderado ${ }^{17-19}$. Reforçando estes resultados, Schmidt et al. ${ }^{20}$ também encontraram baixa prevalência de atividade física moderada, mesmo em gestantes com um maior nível educacional e melhores condições socioeconômicas, em estudo realizado com mulheres de Massachusetts. O estudo realizado por Silva ${ }^{12}$ também constatou uma alta prevalência do padrão leve e sedentário, atingindo $80 \%$ das mulheres. Este fato pode ser explicado pela redução nas atividades com maior índice durante a evolução da gestação, como é o caso das caminhadas, subir escadas, natação, corrida, entre outras ${ }^{6}$.

Observando-se os grupos de atividades verificou-se uma maior média do TTSP relacionados à inatividade, seguidas pelas atividades domésticas, laborais e caminhada. Chama a atenção para a baixa média do TTSP gasto com caminhada, mesmo sendo consideradas neste grupo todas as caminhadas realizadas pelas gestantes, como levar criança para escola, fazer compras, caminhar para o trabalho, entre outras. Outro fator que merece destaque foi a ausência da prática de esportes entre estas mulheres. Portanto, atividades que requerem um maior gasto energético não eram praticamente executadas pelas gestantes. Domingues et al. ${ }^{8}$, em estudo de base populacional realizado em Pelotas, avaliando 4.471 gestantes, encontraram uma prevalência baixa de gestantes que praticavam atividade física, equivalendo a $10,4 \%$ das mulheres no início da gestação, 8,5\% no segundo trimestre e $6,5 \%$ no terceiro, sendo a caminhada responsável por $77,5 \%$ da atividade física realizada. Estudo transversal conduzido na Jordânia ${ }^{21}$, em 2005, acompanhando 400 gestantes na $20^{\mathrm{a}}$ semana, verificou que as atividades domésticas $\mathrm{e}$ subir escadas foram as principais atividades relatadas. Observa-se, portanto, que, apesar das diferenças culturais, o comportamento das mulheres durante a gestação em relação à atividade física são semelhantes.

Se por um lado observou-se uma redução nas atividades que requerem um maior gasto energético como visto acima, por outro lado a inatividade física, aqui representada principalmente por assistir televisão, além de ter obtido a maior média de TTSP, manteve um padrão estável com a evolução da gestação. Este fato pode ter contribuído para o padrão sedentário prevalente neste grupo de gestantes estudadas. De maneira semelhante ao nosso estudo, Bicalho ${ }^{10}$ verificou em estudo de coorte realizado no Rio de janeiro, que dentre as atividades cotidianas a de maior prevalência era assistir televisão, fato importante na determinação do alto percentual do padrão de atividade física sedentário. Um estudo transversal realizado na Noruega ${ }^{22}$, abrangendo 467 gestantes na $36^{\text {a }}$ semana, detectou um alto percentual de gestantes com atividades sedentárias, com $39 \%$ assistindo televisão ou lendo mais de quatro horas por dia.

Revisando os bancos de dados Medline, Lilacs/Scielo e a Biblioteca Cochrane com os termos "pregnancy and exercise", "pregnancy and physical activity", foram encontrados apenas dezoito estudos sobre padrão de atividade física e gravidez, porém estes foram realizados tanto em países desenvolvidos, a exemplo dos Estados Unidos ${ }^{17}$, Noruega $^{22}$, Reino Unido ${ }^{16}$, como em países em desenvolvimento, como o Brasil e Jordânia $^{21}$. Dentre os realizados no Brasil ${ }^{8,10,12}$, em diversas regiões (Sul, Sudeste e Nordeste), observa-se que, a despeito das marcantes diferenças culturais e socioeconômicas nas populações estudadas, verificou-se em todos a tendência à redução do nível de atividade física no final do terceiro trimes- 
tre de gestação. Ao comparar com nossos achados, observa-se que a queda do padrão é maior. Este fato talvez possa ser explicado pelo baixo percentual de mulheres que se encontravam trabalhando no início da gestação nesta coorte (35,7\%), além da baixa prevalência de mulheres que praticavam exercício físico, sendo a caminhada a única modalidade de exercício observada, uma vez que nenhuma destas mulheres realizava qualquer tipo de esporte. Outro fator que pode justificar esta maior queda do padrão com a evolução da gestação é a falta de estímulo para a prática de atividade física por parte da equipe responsável pelo pré-natal. Devido ao pequeno tamanho amostral, o presente estudo não tem poder suficiente para afirmar a associação entre estes fatores e a acentuada queda do padrão de atividade física com a evolução da gestação.

Outra limitação que pode ser considerada neste estudo foi o uso de questionário para avaliar o padrão de atividade física, uma vez que poderia se constituir num viés de informação por se tratar de população de baixa escolaridade. Na literatura encontram-se várias técnicas de mensuração deste padrão, podendo os instrumentos, de forma geral, ser divididos em os que utilizam as informações oriundas dos sujeitos (questionários, entrevistas e diários, a partir dos quais pode ser calculado o gasto energético) e os marcadores fisiológicos e sensores de movimento (pedômetro, acelerômetro, entre outros) ${ }^{23}$. A escolha deste instrumento de coleta para calculo dos METs foi embasada em artigo que compara a utilização de questionário e de sensores de movimentos para a avaliação do padrão de atividade física, sem diferenças estatisticamente significantes ${ }^{24}$. Além disso, os questionários são amplamente utilizados em estudos nacionais e internacionais.

Contudo, parece ser plausível admitir que a redução do padrão de atividade física ao longo do período gestacional é uma realidade que envolve as gestantes independentemente da sua condição cultural e econômica, porém parece ser mais evidente em gestantes de baixa renda e em sociedades onde as mulheres não são estimuladas para a prática de atividade física. Faz-se necessária a realização de estudos ulteriores, envolvendo um maior número de gestantes, na tentativa de se estimular a melhoria do padrão de atividade física entre as gestantes.

Sugere-se que as gestantes sejam estimuladas a realizar atividade física, porém urge que os profissionais responsáveis pela assistência pré-natal recebam treinamento adequado. $\mathrm{O}$ ideal é que a prática de atividade física ocorra de forma planejada, acompanhada e sistematizada no sentido de modificar o padrão de atividade física das gestantes, de forma a melhorar a qualidade de vida das mulheres durante a gestação. Sabemos, entretanto que ainda estamos longe do ideal, mas estes dados, apesar de baseados em amostra pequena da região de Campina Grande-PB, são concordantes com outros estudos nacionais e, portanto, suscitam inquietações neste sentido, apesar de todas as limitações deste estudo. O Ministério da Saúde vem desenvolvendo programas neste sentido, como o Programa Nacional de Promoção da Atividade Física "Agita Brasil", que estimula a prática de atividade física, de preferência diariamente, porém o programa dá ênfase a segmentos expostos a fatores de risco para doenças crônicas não transmissíveis ${ }^{25}$. Observa-se, desta forma, uma carência de programas voltados para as gestantes.

Um ensaio clínico com o objetivo de avaliar o efeito da caminhada sobre o condicionamento físico e os desfechos gestacionais e perinatais encontra-se atualmente em andamento na cidade de Campina Grande, prevendo-se a inclusão de 150 gestantes.

Conclui-se, portanto, que novos estudos são necessários com a finalidade de detectar se este achado é isolado ou se trata de um fenômeno global e, a partir daí, incluir medidas visando o estímulo de um estilo de vida mais saudável nos cuidados de rotina dedicados as gestantes.

Conflito de interesses: Não há. 


\section{Referências}

1. Cuilin Z, Caren GS, JoAnn EM, Frank BH. A Prospective Study of Pregravid Physical Activity and Sedentary Behaviors in Relation to the Risk for Gestational Diabetes Mellitus. Arch Intern Med 2006; 166: 543-8.

2. Clapp JF, Kim H, Burciu B, Lopez B. Beginning regular exercise in early pregnancy: effect on fetoplacental growth. Am J Obstet Gynecol 2000; 183: 1484-8.

3. Batista DC, Chiara VL, Gugelmin SA, Martins PD. Physical activity and pregnancy: non-athletic pregnant women's health and fetal growth. Rev Bras Saude Mater Infant 2003; 3: 151-8.

4. Matsudo VKR, Matsudo SMM. Atividade física e esportiva na gravidez. In: Tedesco JJ, ed. A grávida. São Paulo: Atheneu; 2000. p. 53-81.

5. Caspersen CJ, Kriska AM, Dearwater SR. Physical activity epidemiology as applied to elderly populations. Baillieres Clin Rheumatol 1994; 8: 7-27.

6. Ainsworth BE, Haskell WL, Whitt MC, Irwin ML, Swartz AM, Strath SJ, et al. Compendium of physical activities: an update of activity codes and MET intensities. Med Sci Sports Exerc 2000; 32: 498-516.

7. Chasan-Taber L, Schimdt MD, Roberts DE, Hosmer D, Markenson G, Freedson PS. Development and Validation of a pregnancy physical activity questionnaire. Med Sci Sports Exerc 2004; 36: 1750-60.

8. Domingues MR, Barros AJD. Atividade física de lazer entre as gestantes da coorte de nascimentos de Pelotas de 2004. Rev Saúde Pública 2007; 41: 173-80.

9. Tanya KS, Michelle AW, I-Min L, Edward ED, Mary LT, David AL. Hypertension 2003; 41: 1273-80.

10. Bicalho, Elton de Souza. Fatores associados à prática de atividade física no primeiro trimestre em uma coorte de gestantes do Município do Rio de Janeiro. [dissertação de mestrado] - Universidade Federal do Rio de Janeiro/ Instituto de Nutrição Josué de Castro; 2006.

11. Takito MY, Benício MH D, Latorre MRDO. Postura materna durante a gestação e sua influência sobre o peso ao nascer. Rev Saúde Pública 2005; 39(3): 325-32.

12. Silva FT. Avaliação do nível de atividade física durante a gestação. Rev Bras Ginecol Obstet 2007 ; 29: 490-500.

13. Takito Monica Yuri, Neri Lenycia de Cassya Lopes, Benício Maria Helena D’Aquino. Avaliação da reprodutibilidade e validade de questionário de atividade física para gestantes. Rev Bras Med Esporte 2008; 14: 132-8.
14. Christófalo C, Martins AJ, Tumelero S. A prática de exercício físico durante o período de gestação. Revista Digital: Buenos Aires; 2003.

15. American College of Obstetricians and Gynecologists [ACOG]. Exercise during pregnancy and the postpartum period. ACOG Technical Bulletin 1994; Washington DC. p.189.

16. Rousham EK, Clarke PE, Gross H. Significant changes in physical activity among pregnant women in the UK as assessed by accelerometry and self-reported activity. Eur J Clin Nutr 2006; 60: 393-400.

17. Orr ST, James SA, Garry J, Prince CB, Newton ER. Exercise and pregnancy outcome among urban, lowincome, black women. Ethn Dis 2006; 16: 933-7.

18. Hatch MC, Shu XO, Mclean DE. Maternal exercise during pregnancy, physical fitness, and fetal growth. Am J Epidemiol 1993; 137: 1105-1114.

19. ACOG. Committee on obstetric. Exercise during pregnancy and the postpartum period. Pratice n. 267. Am Col Obstet Gynecol 2002; 99: 171-3.

20. Schmidt MD, Penelope P, Patty SF, Glenn MMD, ChasanTaber L. Physical Activity Patterns during Pregnancy in a Diverse Population of Women.J Womens Health 2006; 15: 909-18.

21. Gharaibeh M, Al-Ma'aitah R, Al Jada N. Lifestyle practices of Jordanian pregnant women, International Council of Nurses 2005; 52: 92-100.

22. Haakstad LAH, Nanna V, Tore H. Physical activity level and weight gain in a cohort of pregnant Norwegian women, Acta Obstet Gynecol Scand 2007, 86: 559-64.

23. Reis RS, Petroski EL, Lopes AS. Medidas da atividade física: revisão de métodos. Rev Bras Cineantrop Desempenho Hum 2000; 2: 89-96.

24. Lindseth G, Vari P. Measuring physical activity during pregnancy. West J Nurs Res 2005; 27: 722-34.

25. Ministério da Saúde. Programa Nacional de Promoção da Atividade Física "Agita Brasil": atividade física e sua contribuição para a qualidade de vida. Rev Saúde Pública 2002; 36(2): 254-6.

Recebido em: 27/06/08 Versão final reapresentada em: 11/11/08 Aprovado em: 17/12/08 